\title{
Procesos de transferencia en conceptos de probabilidad: Una propuesta didáctica
}

\author{
María Dolores González Quezada \\ Instituto Tecnológico de Ciudad Juárez \\ mdolores@nmsu.edu \\ Janette Olivia Chávez Prieto \\ Universidad Autónoma de Ciudad Juárez \\ jachavez@uacj.mx \\ Juan Ernesto Chávez Pierce \\ Universidad Autónoma de Ciudad Juárez \\ juchavez@uacj.mx
}

\section{Resumen}

Esta investigación se centra en el análisis de transferencia de aprendizaje ante cambios de contexto en situaciones que involucran conceptos de probabilidad. La investigación se condujo con dos grupos de estudiantes de nivel superior (experimento y control), con la finalidad de explorar los efectos en el entendimiento y en la transferencia de conceptos que produce la implementación de una propuesta didáctica. Está propuesta didáctica se fundamenta en la visualización de situaciones de contexto en el plano concreto. Esto a través de la utilización de material manipulativo como recurso didáctico (urnas, ruletas, barajas y dados). En el grupo control se trabajó bajo una instrucción de corte tradicionalista y en el grupo experimento se implementó la propuesta didáctica. Los elementos de evaluación durante el proceso cognitivo fueron los mismos para ambos grupos. Un examen de salida ayudó a evaluar la efectividad en el aprendizaje de la secuencia didáctica y a encontrar evidencia de transferencia. Las observaciones muestran que la secuencia didáctica posiblemente estimuló la asignación de significados y promovió la transferencia de conceptos mediante la utilización de recursos didáctico-visuales.

\section{Palabras clave}

Transferencia de aprendizaje, probabilidad, recursos didácticos, problemas de entendimiento.

\section{Introducción}

En los últimos años los investigadores en el área de matemática educativa se han preocupado por mejorar los resultados obtenidos en los procesos de enseñanza y aprendizaje. Estos procesos y sus distintos aspectos ontológicos, epistemológicos, cognitivos e instruccionales se han intentado analizar y explicar desde diversos enfoques teóricos $\mathrm{y}$ metodológicos. En este sentido, en didáctica de la probabilidad se ha puesto de manifiesto el notable incremento de materiales didácticos, software educativo, investigaciones, revistas, reuniones y congresos sobre la enseñanza y el aprendizaje de esta disciplina. La probabilidad como parte esencial de las 
RECIE. Revista Electrónica Científica de Investigación Educativa Vol. 1, núm. 1, enero-diciembre 2012, pp. 157-167.

matemáticas se encarga del estudio de los fenómenos aleatorios. Dichos fenómenos están presentes en innumerables situaciones de la vida cotidiana y tienen la característica de generar resultados $\mathrm{u}$ observaciones que no son susceptibles de predecirse con certeza, aunque estos se estudien repetidamente bajo las mismas condiciones. Los términos azar $y$ estocástico también se usan comúnmente para hacer referencia al carácter imprevisible de estos fenómenos. Una formación básica en probabilidad permite interpretar eventos de la vida cotidiana que se producen en contextos probabilísticos y de esta forma tomar decisiones en situaciones de incertidumbre. Es por esto que la probabilidad ha formado parte de la formación básica en los estudios universitarios. Una de las mayores dificultades a la que los estudiantes de las carreras de ingeniería de la universidad Autónoma de Ciudad Juárez (UACJ) se enfrentan en el aprendizaje de la teoría de la probabilidad, es la falta de aprendizaje significativo de los conceptos. Una de las posibles causas podría ser el método de enseñanza tradicional utilizado por la mayoría de los instructores (Flores, Chávez, Luna, González, González y Hernández, 2008). Por tal motivo, este aprendizaje se ha convertido en fuente de constante preocupación entre el profesorado y el campo de los conceptos relativos al azar y la probabilidad en una de las áreas emergentes de investigación en la enseñanza y el aprendizaje de las matemáticas (Barragués, Guisasola y Morais, 2005). No obstante, la cantidad de investigaciones sobre la didáctica de la probabilidad todavía es muy escasa en comparación con las existentes en otras ramas de las matemáticas $y$ en consecuencia no se conocen aún cuales son las principales dificultades de los alumnos en el entendimiento de muchos conceptos importantes (Batanero, 2001).

\section{Problema de investigación}

La mayoría de los estudiantes que ingresan a las universidades, lo hacen con conocimientos casi nulos en nociones básicas de probabilidad o con creencias e intuiciones incorrectas de estas nociones que les dificulta la comprensión de los diferentes tópicos de esta disciplina (Carrera, 2007). Aunado a esto, se tiene que la probabilidad que se enseña en los cursos regulares se da de una forma poco natural, basada en una instrucción de corte tradicionalista (Calva, 2005). Es por esto que el objetivo principal de esta investigación es valorar la efectividad de una propuesta didáctica fundamentada en la visualización de situaciones de contexto en el plano concreto en base a la utilización de material manipulativo como recurso didáctico (urnas, ruletas, barajas y dados). Con esta propuesta se tiene la intención de mejorar el entendimiento conceptual de los estudiantes de la materia de Probabilidad y estadística en el Instituto de Ingeniería y Tecnología de la UACJ y promover la transferencia de conceptos probabilísticos entre diversos contextos. Se pretende explorar si existe evidencia de transferencia de conceptos y medir la ganancia como indicador de la efectividad del aprendizaje. Esto a partir de los resultados arrojados por los instrumentos de evaluación diseñados y posteriormente administrados a dos grupos de estudiantes: grupo experimento y grupo control. En el grupo experimento se implementó la propuesta didáctica y en el grupo control se trabajó bajo una instrucción de corte tradicionalista. La pregunta de investigación es: ¿Qué efectos en la transferencia de conceptos de 
probabilidad produce en los estudiantes una propuesta didáctica fundamentada en la visualización de situaciones de contexto en el plano concreto?

\section{Fundamentación teórica}

Esta investigación se fundamenta en la teoría de la transferencia del aprendizaje. La transferencia como parte esencial de los procesos del aprendizaje, ha sido definida como el proceso mediante el cual los conocimientos (conceptos, operaciones, estrategias, actitudes y habilidades) adquiridos en un contexto se utilizan en otros contextos diferentes. De este modo, los aprendizajes no quedan anclados a una situación específica y pueden ser utilizados en nuevas condiciones y escenarios (Carpintero, 2002). Al respecto, Mayer y Wittrock (1996) señalan que siempre que algo se aprendió previamente afecta el aprendizaje actual o cuando la solución de un problema anterior afecta la manera en la que se resuelve un problema nuevo, ocurre transferencia. El aprendizaje que se adquiere principalmente en la escuela se explica basado en el supuesto de que lo que se aprende en una ocasión facilitará el buen desempeño en situaciones relacionadas (Beltrán, Moraleda, García-
Alcañiz, Calleja y Santiuste, 1995). En este sentido, McKeough, Lupart y Marini, 1995 enuncian que una de las principales metas en la educación es que los estudiantes utilicen en el área de su desempeño laboral los conocimientos adquiridos en las instituciones educativas. Debido a esto, diversos investigadores han definido la transferencia del conocimiento como el fin último de la educación (McKeough, Lupart y Marini, 1995). Por lo tanto, la transferencia es un concepto clave en la teoría de la enseñanza y el aprendizaje.

Para Hoffding (1892) el proceso de transferencia de un conocimiento o concepto desde una situación de aprendizaje $A$ hasta su respuesta observable $B$ puede ser representado por medio del recuerdo (Figura 1). Según Hoffding recordar es cuando el evento $A$ activa de nuevo a su representación interna $a$, la cual es representada por una respuesta interna $b$ que da como resultado la aparición del evento $B$ previamente aprendido. La cadena de Hoffding puede ser caracterizada como $A-a-b-B$ en donde la idea central es encontrar un estímulo didáctico que permita activar la respuesta interna $b$ cuando $A$ no es idéntica a su forma inicial, sino una variante $A^{\prime}$.

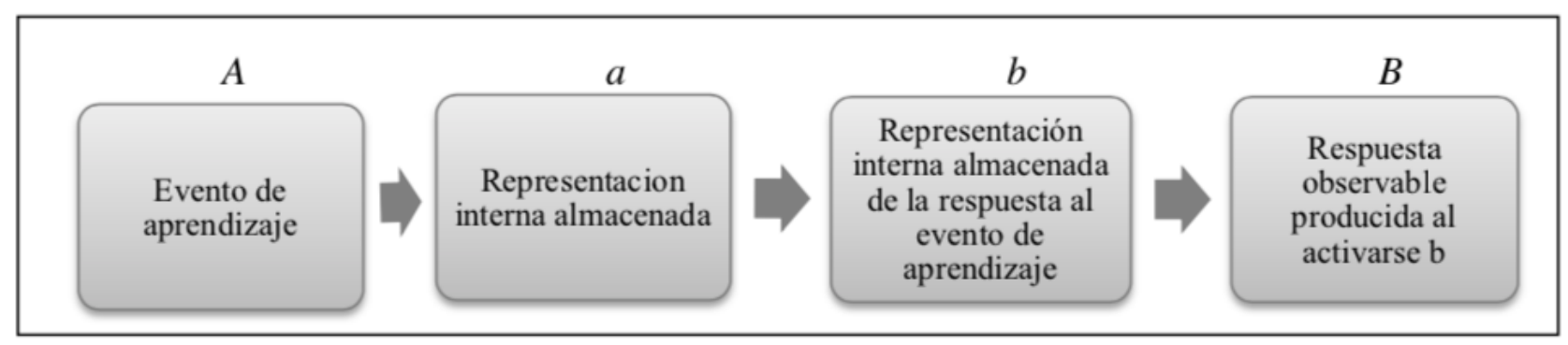

Figura 1. Cadena de Hoffding para representar la transferencia de un evento A con una respuesta observable B.

\section{Descripción de la población}

La investigación se condujo con 47 estudiantes de dos grupos (grupo E y grupo I) de la materia de Probabilidad y estadística del Instituto de Ingeniería y Tecnología de la Universidad Autónoma de 
Ciudad Juárez (UACJ). En el grupo experimento (Grupo F) y en el grupo control (Grupo I) participaron 28 y 19 estudiantes respectivamente. La materia de Probabilidad y estadística es obligatoria para todas las carreras de ingeniería de la UACJ y por lo tanto, ambos grupos estuvieron integrados por estudiantes adscritos a diversos programas de ingeniería. Actualmente los tópicos de probabilidad incluidos en los contenidos temáticos de este curso, son abordados en dos sesiones semanales de 120 minutos cada una. Esto bajo una instrucción prácticamente de corte tradicionalista. El libro de texto es Probabilidad \& Estadística para ingeniería y ciencias (Walpole, Myers, Myers y Ye, 2007).

\section{Metodología}

Esta investigación de carácter cualitativo y cuantitativo se realizó durante el semestre Agosto-diciembre del 2011. Durante toda la investigación se eliminó la variable instructor dado que éste fue el mismo para los dos grupos de trabajo: Grupo experimento y grupo control. Los elementos de evaluación (examen de entrada y salida) durante el proceso cognitivo fueron los mismos para ambos grupos.

\section{Examen de entrada}

Se diseñó y administró en ambos grupos un examen de entrada (antes de la instrucción), con el fin de evaluar conocimientos previos de probabilidad. En este examen de incluyeron 20 reactivos de diferente tipo: comparación, orden, análisis de enunciados, y solución de contingencias (Kanim,1999) diseñados bajo el régimen de opción múltiple. En todos los reactivos se les pidió a los estudiantes la justificación a su respuesta. Los problemas de cada reactivo fueron formulados en pares con la finalidad de encontrar en los estudiantes, evidencia de transferencia de conceptos probabilísticos. Cada par corresponde a problemas análogos (aluden al mismo concepto o estructura matemática) pero sus contextos son distintos. Un ejemplo de un par de reactivos se muestra en la Figura 2. 
Problema 1. Si se lanzan dos dados, uno gris y uno blanco, con caras marcadas del 1 al 6 (figura de la derecha). La probabilidad de que la suma de las caras que caen hacia arriba sea 11 o ambas caras muestren el mismo número es...

(a) Mayor que 0 pero menor que $2 / 9$

(b) Exactamente $2 / 9$

(c) Mayor que $2 / 9$ pero menor que 1.0

(d) Mayor o igual que 1.0

Justifique su respuesta

Problema 9. La figura de la derecha muestra dos bolsas con tarjetas. La bolsa \#1 contiene 7 tarjetas blancas marcadas con números del 1 al 7 y la bolsa \#2 contiene 8 tarjetas negras marcadas con números del 1 al 8 . Si se selecciona una tarjeta de cada bolsa y $E$ es el evento para el cual la suma de los números de las tarjetas que se sacan de las bolsas es 3 o 4 . La probabilidad de que ocurra el evento $E$ es...
(a) $0<\mathrm{P}(E)<5 / 15$
(b) $\mathrm{P}(E)=5 / 15$
(c) $5 / 15<\mathrm{P}(E)<1.0$
(d) $\mathrm{P}(E) \geq 1.0$

Justifique su respuesta

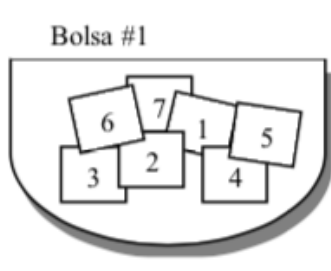

Bolsa \#2

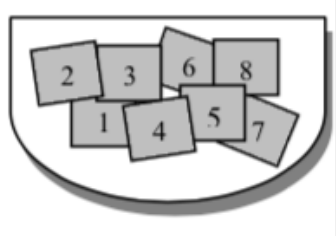

Figura 2. Par de problemas que aluden al uso del mismo concepto (regla de la suma para eventos mutuamente excluyentes).

\section{Propuesta didáctica}

Dado que la enseñanza de los contenidos incluidos en los programas de la mayoría de los cursos regulares de probabilidad se da de forma poco natural y bajo una instrucción de corte tradicionalista (Calva, 2005), es necesario desarrollar e implementar nuevas estrategias didácticas para la enseñanza y el aprendizaje de dichos contenidos. Por tal motivo se procedió al diseño y posterior implementación de una secuencia didáctica (2 actividades) para la enseñanza y el aprendizaje de algunos tópicos de Probabilidad: Concepto clásico de la probabilidad, eventos y espacio muestral (Primera actividad) y reglas de la probabilidad (Segunda Actividad). Esta secuencia didáctica se fundamentó en la visualización de situaciones de contexto en el plano concreto a través de la utilización de material manipulativo como recurso didáctico (urnas, ruletas, baraja y dados). En el grupo experimento se implementó la secuencia didáctica $(2$ sesiones de 60 minutos cada una para cada actividad) y en el grupo control se trabajó bajo una instrucción de corte tradicionalista (4 sesiones de 60 minutos cada una). La secuencia didáctica se realizó de forma grupal (equipos de 4 a 5 estudiantes) con el fin de fomentar el aprendizaje colaborativo cuyo objetivo es inducir a los participantes a la exploración, discusión, negociación y debate (Hsu,2002). Al finalizar cada actividad se realizó una retroalimentación entre todos los equipos y el profesor. Las dos actividades fueron el único material de aprendizaje al que se expusieron los estudiantes. En la Figura 3 se muestra un ejemplo de uno de los ejercicios incluidos en la primera actividad de la secuencia didáctica. 


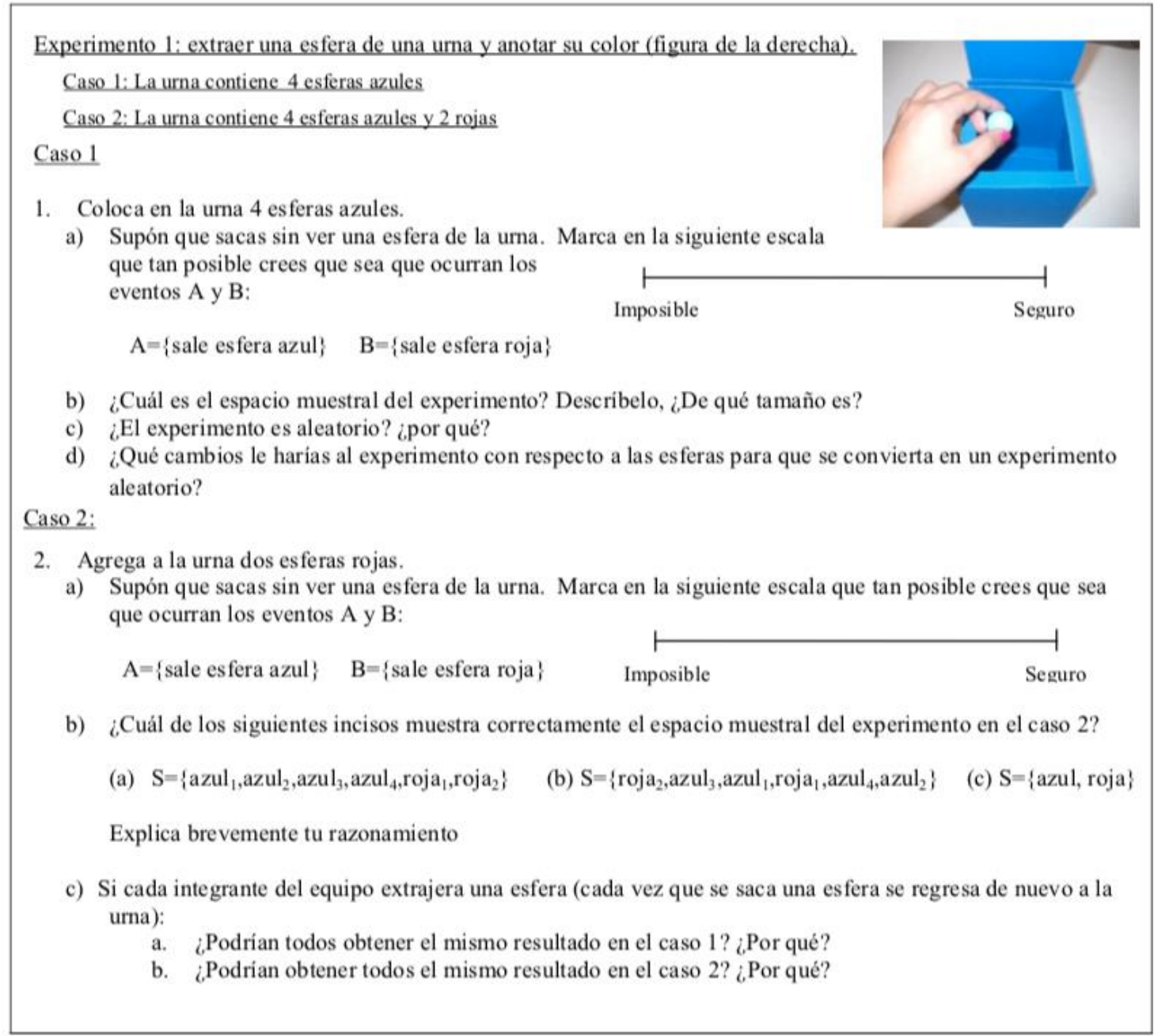

Figura 3. Parte del experimento 1 incluido en la primera actividad de la secuencia didáctica (conceptos de experimento determinístico y estocástico).

\section{Examen de salida y transferencia de aprendizaje}

Un examen de salida (el mismo que el de entrada) ayudó a evaluar la efectividad del aprendizaje en ambos grupos de trabajo y a encontrar evidencia de transferencia de conceptos. Esto en base al modelo desarrollado por González y Kanim (2008). Este modelo (Figura 4) permite observar si existe evidencia de transferencia de conocimiento en pares de problemas que requieren el uso del mismo concepto en dos contextos diferentes. De acuerdo al modelo, las respuestas correctas e incorrectas del primer contexto se ubican de forma horizontal y las respuestas correctas e incorrectas del segundo contexto se ubican de forma vertical. La intersección de las respuestas correctas en ambos contextos corresponde al bloque azul en la parte superior izquierda. Los autores asumen que los estudiantes que se ubican en esta área presentan evidencia del uso del mismo concepto para contestar correctamente ambas preguntas. Los dos bloques rojos corresponden al número de estudiantes que no son consistentes en sus respuestas al cambiar de un contexto a otro. En este caso se asume que los 
estudiantes no presentan evidencia de transferencia. La intersección de respuestas incorrectas en ambos contextos se localiza en la parte inferior derecha (bloque verde). Lo anterior se podría asumir como una transferencia negativa, es decir, es posible que los estudiantes hayan utilizado el mismo concepto erróneo para responder incorrectamente las dos preguntas. A partir de los razonamientos mostrados por los estudiantes en la resolución de los problemas, se estableció una clasificación de las estrategias de resolución. Las categorías de clasificación se establecieron tomando en cuenta los procesos realizados y las herramientas utilizadas por los estudiantes.

\section{$\underline{\text { Segundo contexto }}$}

\section{Correcto Incorrecto}

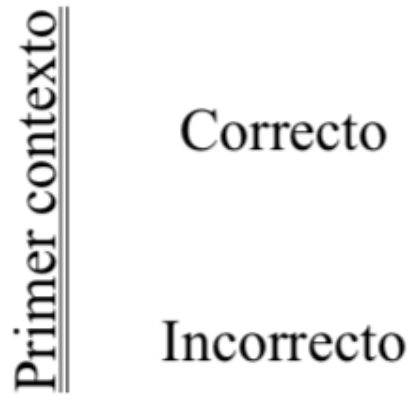

\begin{tabular}{|c|c|}
\hline $\begin{array}{c}\text { Probable } \\
\text { transferencia }\end{array}$ & $\begin{array}{c}\text { No existe } \\
\text { evidencia de } \\
\text { transferencia }\end{array}$ \\
\hline $\begin{array}{c}\text { No existe } \\
\text { evidencia de } \\
\text { transferencia }\end{array}$ & $\begin{array}{c}\text { Posible } \\
\text { transferencia } \\
\text { negativa }\end{array}$ \\
\hline
\end{tabular}

Figura 4. Modelo para encontrar evidencia de transferencia.

\section{Ganancia entrada-salida}

A partir de los exámenes de entrada y salida administrados en ambos grupos, se calculó la ganancia normalizada, Hake (1998) como indicador de la efectividad de la instrucción en el aprendizaje. Esta ganancia normalizada es una medida de la razón entre el incremento del número de aciertos en el examen de salida con respecto al examen de entrada y el máximo incremento posible. La ganancia normalizada se calculó con la siguiente ecuación:

$$
G=\frac{\% \text { salida }-\% \text { entrada }}{100-\% \text { entrada }}
$$

\section{Discusión de resultados}

Las observaciones muestran que ambos grupos mostraron mejores resultados en el examen de salida, es decir, después de la enseñanza, independientemente del tipo de instrucción (tradicional o a través de recursos manipulables). La ganancia normalizada como medida de la razón entre el incremento del número de aciertos en el examen de salida con respecto al examen de entrada y el máximo incremento posible fue de 0.53 en el grupo experimento y 0.50 en el grupo control. Según la propuesta de Hake (1998) la efectividad de la instrucción se categoriza en rangos de ganancia normalizada baja $(\mathrm{g}<0.3)$ media $(0.3<\mathrm{g}<0.7)$ y alta $(\mathrm{g}>0.7)$. Por lo tanto, se 
obtuvo una ganancia normalizada media en ambos grupos.

La tabla 1 muestra el porcentaje de estudiantes que muestran evidencia de transferencia positiva, negativa y falta de transferencia. En 8 de los 10 pares de problemas, las respuestas de los estudiantes del grupo experimento fueron consistentes entre los dos contextos (6 corresponden a transferencia positiva), por lo tanto, en 2 de los 10 pares de problemas las respuestas fueron inconsistentes entre los dos contextos (falta de transferencia). En el grupo control en 8 de los 10 pares de problemas, las respuestas de los estudiantes fueron consistentes entre los dos contextos (5 corresponden a transferencia positiva).

Tabla 1. Porcentaje de estudiantes con evidencia de transferencia (positiva y negativa) y falta de transferencia en cada par de problemas (mismo concepto- contextos diferentes).

\begin{tabular}{|c|c|c|c|c|c|c|c|}
\hline \multirow[t]{2}{*}{ Concepto } & \multirow[t]{2}{*}{$\begin{array}{l}\text { Número de } \\
\text { reactivo }\end{array}$} & 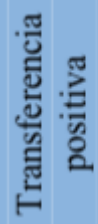 & 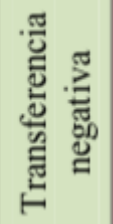 & 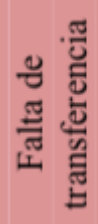 & 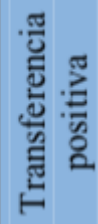 & 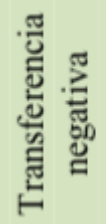 & 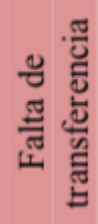 \\
\hline & & \multicolumn{3}{|c|}{ Grupo experimento } & \multicolumn{3}{|c|}{ Grupo control } \\
\hline $\begin{array}{l}\text { Regla de la suma eventos } \\
\text { mutuamente excluyentes }\end{array}$ & $\begin{array}{l}\text { Reactivo } 1 \\
\text { Reactivo } 9\end{array}$ & $21 \%$ & $18 \%$ & $61 \%$ & $26 \%$ & $58 \%$ & $16 \%$ \\
\hline $\begin{array}{l}\text { Regla de la suma eventos no } \\
\text { excluyentes }\end{array}$ & $\begin{array}{l}\text { Reactivo } 2 \\
\text { Reactivo } 10\end{array}$ & $21 \%$ & $39 \%$ & $39 \%$ & $21 \%$ & $32 \%$ & $47 \%$ \\
\hline Regla del complemento & $\begin{array}{l}\text { Reactivo } 3 \\
\text { Reactivo } 6\end{array}$ & $89 \%$ & $4 \%$ & $7 \%$ & $84 \%$ & $0 \%$ & $16 \%$ \\
\hline $\begin{array}{l}\text { Regla de la multiplicación } \\
\text { eventos dependientes. }\end{array}$ & $\begin{array}{l}\text { Reactivo } 4 \\
\text { Reactivo } 14\end{array}$ & $25 \%$ & $21 \%$ & $54 \%$ & $47 \%$ & $16 \%$ & $37 \%$ \\
\hline $\begin{array}{l}\text { Regla de la multiplicación } \\
\text { eventos independientes }\end{array}$ & $\begin{array}{l}\text { Reactivo } 5 \\
\text { Reactivo } 12\end{array}$ & $54 \%$ & $32 \%$ & $14 \%$ & $58 \%$ & $0 \%$ & $42 \%$ \\
\hline $\begin{array}{l}\text { Regla de la multiplicación } \\
\text { eventos independientes }\end{array}$ & $\begin{array}{l}\text { Reactivo } 7 \\
\text { Reactivo } 15\end{array}$ & $68 \%$ & $0 \%$ & $32 \%$ & $84 \%$ & $0 \%$ & $16 \%$ \\
\hline $\begin{array}{l}\text { Regla de la multiplicación } \\
\text { eventos dependientes. }\end{array}$ & $\begin{array}{l}\text { Reactivo } 8 \\
\text { Reactivo } 19\end{array}$ & $32 \%$ & $32 \%$ & $36 \%$ & $5 \%$ & $63 \%$ & $32 \%$ \\
\hline $\begin{array}{l}\text { Regla de la multiplicación } \\
\text { eventos independientes }\end{array}$ & $\begin{array}{l}\text { Reactivo } 11 \\
\text { Reactivo } 17\end{array}$ & $71 \%$ & $11 \%$ & $18 \%$ & $21 \%$ & $21 \%$ & $58 \%$ \\
\hline $\begin{array}{l}\text { Regla de la suma y de la } \\
\text { multiplicación. }\end{array}$ & $\begin{array}{l}\text { Reactivo } 13 \\
\text { Reactivo } 20\end{array}$ & $50 \%$ & $4 \%$ & $46 \%$ & $32 \%$ & $11 \%$ & $58 \%$ \\
\hline $\begin{array}{l}\text { Regla de la multiplicación } \\
\text { eventos independientes }\end{array}$ & $\begin{array}{l}\text { Reactivo } 16 \\
\text { Reactivo } 18\end{array}$ & $71 \%$ & $11 \%$ & $18 \%$ & $84 \%$ & $0 \%$ & $16 \%$ \\
\hline
\end{tabular}

En la Figura 5 se puede ver un ejemplo de análisis de transferencia de conceptos bajo el modelo propuesto por González y Kanim
(2008) para un par de problemas (problema 1 y 9). La tabla 2 muestra algunos razonamientos de estudiantes 
cuyas respuestas sugieren transferencia positiva, falta de transferencia y transferencia negativa en este par de problemas. Un análisis semejante se realizó con cada par de problemas con el objeto de clasificar los razonamientos de los estudiantes de acuerdo a las estrategias de resolución que manifestaron los estudiantes en cada problema.

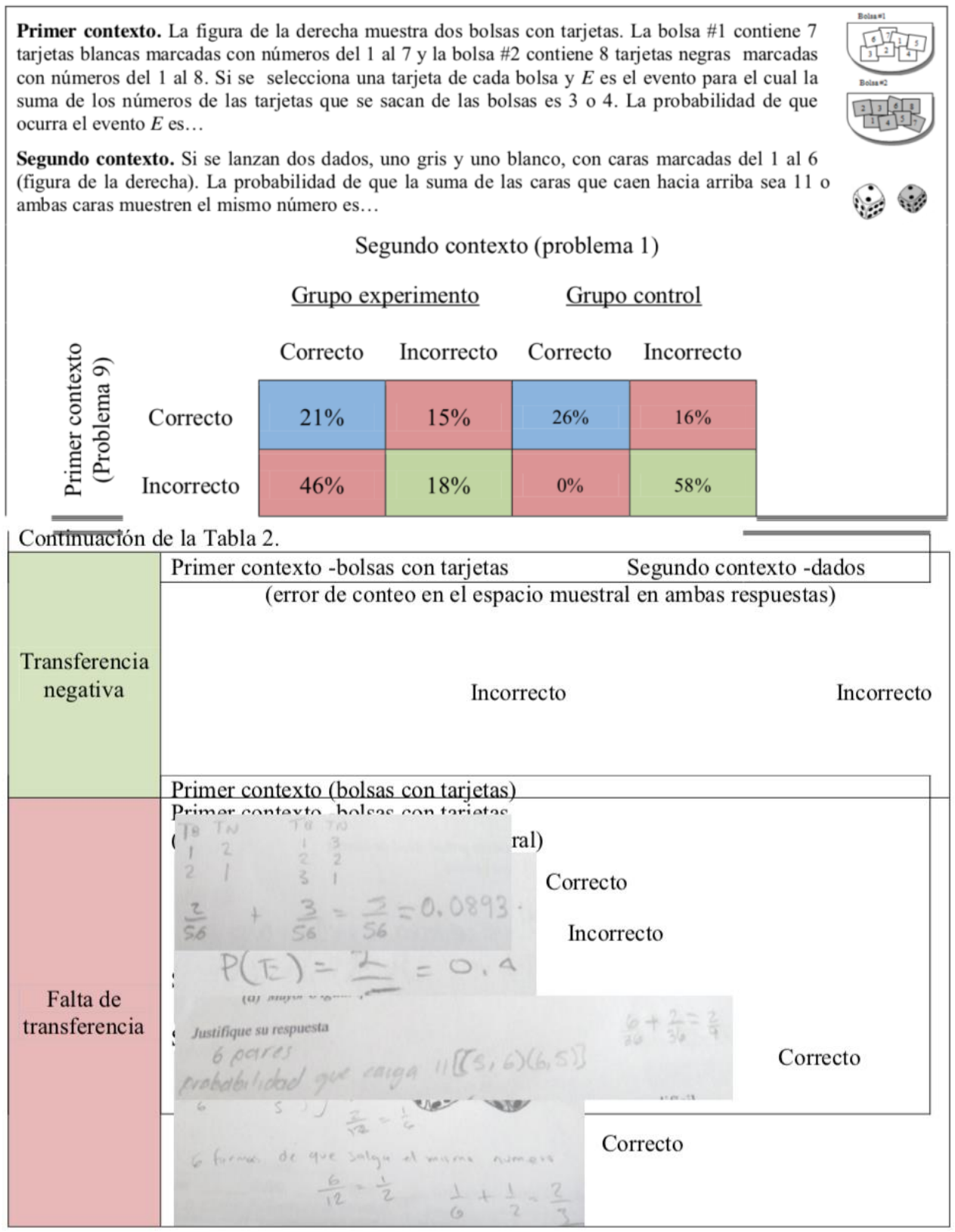

Figura 5. Ejemplo de análisis de transferencia de conceptos entre dos contextos (Problema 1 y 9). Tabla 2. Razonamientos que sugieren transferencia positiva, transferencia positiva y falta de transferencia.

\section{Conclusiones}

A través de la investigación se pudieron

Procesos de transferencia en conceptos de probabilidad: Una propuesta didáctica conocer problemas de entendimiento, dificultades y errores que se presentan con 
RECIE. Revista Electrónica Científica de Investigación Educativa Vol. 1, núm. 1, enero-diciembre 2012, pp. 157-167.

cierta regularidad entre los estudiantes como producto de la propia situación del problema $\mathrm{y} / \mathrm{o}$ concepto probabilístico involucrado. Se esperaba que los estudiantes del grupo experimento contestaran mejor y mostraran un mayor desarrollo de la habilidad para transferir conceptos de un contexto a otro contexto. En este sentido, dado que la ganancia como indicador de la efectividad del aprendizaje en el grupo experimento fue de 0.53 podríamos pensar que la propuesta didáctica posiblemente estimuló la asignación de significados mediante la utilización de recursos didáctico-visuales. No obstante, en el grupo control la ganancia fue de 0.50 por lo que se observaron resultados semejantes. En el grupo experimento se observó un ligero incremento en el número de pares de reactivos con transferencia positiva, por lo que también los resultados fueron semejantes. Es posible que el uso exclusivo de los recursos didáctico-visuales en el plano concreto (representación enactiva) y la falta de explicación directa al grupo experimento por parte del instructor fueron factores de la semejanza de resultados entre ambos grupos. No obstante, en el proceso de aprendizaje de la Probabilidad, la motivación es un factor determinante para el éxito que pueda tener el estudiante. La utilización de recursos didácticos como los que aquí se presentan deben estar encaminados a influir en aquellos estudiantes con bajo nivel motivacional, despertando su interés y la necesidad de alcanzar su aprendizaje. Es por esto que tal vez deban ser utilizados como complemento a otros métodos de enseñanza. Este tipo de propuestas, como es señalado por Evans (2010), logra una transformación de la realidad, la que se da en el mismo proceso de su ejecución, sobre todo porque transforma a todos los sujetos involucrados, ya que al ser participantes activos interiorizan y tiene la oportunidad de valorar otras vivencias distintas a las experimentadas en contextos tradicionales.

\section{Agradecimientos}

Agradecemos al Dr. Stephen Kanim de la Universidad Estatal de Nuevo México, NMSU, por el desarrollo del modelo para encontrar evidencia de transferencia en pares de preguntas que fue utilizado en esta investigación.

\section{Referencias}

Barragués, J. I., Guisasola, J., y Morais, A. (2005). Concepciones de los Estudiantes de Primer Ciclo de Universidad. Educación Matemática, 17 (1), 55-85.

Batanero, C. (2001). Didáctica de la Estadística. Grupo de Investigación en Educación Estadística. Departamento de Didáctica de las Matemáticas. Universidad de Granada. Recuperado el 6 de septiembre de 2011de http://www.ugr.es/ batanero/Pr oyectos.htm

Beltrán. J., Moraleda, M., García-Alcañiz, E., Calleja, F. G. y Santiuste, V. (1995). Psicología de la educación. Madrid: EUDEMA.

Calva, L. (2005). Consideraciones sobre algunos conceptos básicos de la probabilidad. Tesis de Licenciatura no publicada, Universidad Nacional Autónoma de México.

Carpintero, E. (2002). El proceso del transfer: Revisión y nuevas perspectivas. Revista de psicología y psicopedagogía 1(1),69-95.

Carrera, E. (2002). Teaching statistics in secondary school. An overview: 
From the curriculum to reality. En B. Phillips (Ed.), Proceedings of the Sixth International Conference on Teaching of Statistics. Ciudad del Cabo: IASE.

Evans, E. (2010). Orientaciones metodológicas para la investigación-acción: propuesta para la mejora de la práctica pedagógica. Lima: Ministerio de Educación. Dirección de Investigación, Supervisión y Documentación.

Flores, S., Chávez J. E., Luna, J., González, M. D., González, M. V. y Hernández, A. A. (2008). El aprendizaje de la física y las matemáticas en contexto. Cultura, ciencia y tecnología, 5(24), 19-24.

González, M. D. (2008, enero). Student Use of Mechanic Knowledge in Electrostatic. Ponencia presentada en la reunión anual de invierno de la AAPT, Baltimore, Estados Unidos.

Hake, R. R. (1998). Interactiveengagement vs traditional methods: A six-thousand student survey of mechanics test data for introductory physics courses.
American Journal of Physics, 66, 6474.

Hoffding, H. (1892). Outlines of psychology, London: McMillan. Kanim, S. (1999). An investigation on student difficulties in qualitative and quantitative problem solving. Tesis de doctorado no publicada, University of Washington. Estados Unidos.

Mayer, R. E., y Wittrock, M. C. (1996). Problem-solving transfer. En D. C. Berliner y R. C. Calfee (Eds.), Handbook of Educational Psychology (pp. 47-62). New York: MacMillan Library Reference USA.

McKeough, R., Lupart, J. y Marini, A. (1995). Teaching for transfer: Fostering generalization in learning, Mahwah, NJ: Erlbaum.

Scagnoli, N. I. (2006). El aprendizaje colaborativo en cursos a distancia. Investigación y ciencia, 14 (36), 3947.

Walpole, R. E., Myers, S. L., Myers, K. Y.y Ye, K. (2007). Probabilidad y estadística para ingeniería y ciencias. México: Pearson Educación. 
RECIE. Revista Electrónica Científica de Investigación Educativa

Vol. 1, núm. 1, enero-diciembre 2012, pp. 157-167.

González Quezada, M.D.; Chávez Prieto, J.O.; y Chávez Pierce, J.E. 